\title{
Hemovigilância: utilização das ferramentas de gestão para a qualidade no processo transfusional
}

\author{
Haemovigilance: use of management tools for quality in transfusion process \\ Seguridad de la sangre: empleo de las herramientas de gestión para la calidad del \\ proceso transfusional
}

Ana Carolina Ayami Yoshioka Frazão ${ }^{1}$, Giovana Karina Lima Rolim ${ }^{1}$, Ingrid Pinheiro Pantoja ${ }^{1}$, Lucas Ferreira Martins $^{1}$, Marcos José Risuenho Brito Silva ${ }^{1 *}$, Lívia Félix de Oliveira ${ }^{1}$

\section{RESUMO}

Objetivo: Relatar a experiência da utilização de ferramentas da gestão na promoção de melhorias da qualidade do processo transfusional. Relato da experiência: $O$ presente estudo é baseado na vivência de acadêmicos de enfermagem acerca da utilização de ferramentas da gestão, como a Brainstorming, o diagrama de Ishikawa e o $5 \mathrm{~W} 2 \mathrm{H}$, para definição de um problema e traçar estratégias de melhorias, incluindo a educação permanente dos profissionais que trabalham com hemotransfusão em variadas clínicas de uma instituição. Sendo assim, a utilização de tais ferramentas proporcionou boa receptividade dos profissionais frente à atividade proposta. A educação permanente e a utilização dos ideais do diagrama de Ishikawa ajudaram a fomentar a discussão e identificar as causas, efeitos e ações estratégicas para melhorar os índices de notificação e qualidade do processo transfusional. Considerações Finais: O estudo ressalta a importância da integração entre gestão, assistência e ensino, tendo a educação permanente, como estratégia para minimizar erros e garantir atualização profissional, além de viabilizar a utilização prática dos conhecimentos acadêmicos obtidos na universidade.

Palavras-Chave: Hemovigilância, Gestão em Saúde, Educação Permanente.

\section{ABSTRACT}

Objective: Report the experience of using management tools in promoting improvements in the quality of the transfusion process. Experience Report: The present study is based in the experience of nursing students about the use of the management tools such as Brainstorming, Ishikawa Diagrams and the $5 \mathrm{~W} 2 \mathrm{H}$, to the definition of a problem and to define improvement strategies, including the lifelong education of the professionals working with blood transfusion in several sector of the institution. Thus, the use of such management provided good feedback from the professionals on the proposed activity. The lifelong education and the use of the ideals of the Ishikawa diagram helped fostering discussion and explain the reasons, effects and strategic action to improve the notification indices and the quality of the transfusion process. Final Considerations: The study highlights the importance of integration between management, care and teaching, with lifelong education as strategic to avoid errors and ensure professional upgrading, in addition to practical use of academic knowledge obtained at the university.

Key words: Haemovigilance, Health Management, Life-long Education.

${ }^{1}$ Escola de Enfermagem Magalhães Barata da Universidade do Estado do Pará (EEMB - UEPA), Belém Pará. *E-mail: marcosrisuenho.1@hotmail.com 


\section{RESUMEN}

Objetivo: Informar la experiencia del empleo de herramientas de gestión en la promoción de mejoras de la calidad del proceso transfusional. Relato de la experiencia: Este estudio se basa en la experiencia de académicos de enfermería acerca del empleo de herramientas de gestión como la Brainstorming, el diagrama de Ishikawa y el $5 \mathrm{~W} 2 \mathrm{H}$, con miras a definir un problema y trazar estrategias de mejora, incluyendo la educación continua de los profesionales que trabajan con transfusión sanguínea en diversas clínicas de la institución. Siendo así, el empleo de tales herramientas proporcionó una buena receptividad de los profesionales ante la actividad propuesta. La educación continua y el empleo de los ideales del diagrama de Ishikawa ayudaron a fomentar la discusión e identificar las causas, efectos y acciones estratégicas para mejorar los índices de notificación y calidad del proceso transfusional. Consideraciones finales: El estudio subraya la importancia de la integración entre gestión, asistencia y enseñanza, teniendo la educación continua como estrategia para evitar errores y garantizar la actualización profesional, además de posibilitar el empleo práctico de los conocimientos académicos obtenidos en la universidad.

Palabras Clave: Seguridad de la Sangre, Gestión en Salud, Educación Continua.

\section{INTRODUÇÃO}

A transfusão de hemocomponentes e hemoderivados tem grande relevância e impacto para o sistema de saúde. Existem diversos cuidados e procedimentos preconizados para a qualidade do processo transfusional, os quais se forem seguidos adequadamente podem melhorar ou até mesmo salvar a vida de uma pessoa, além de minimizar significativamente possíveis danos a sua saúde (BRASIL, 2015a).

O serviço de hemoterapia é organizado em rede, tendo diversas etapas que constituem o ciclo do sangue, tais como: a captação de doadores, triagem clínica, coleta de sangue, processamento de sangue em hemocomponentes, análises sorológicas e imuno-hematológicas no sangue do doador, armazenamento, distribuição e transfusão; ressalta-se que a hemovigilância está presente em cada uma das etapas desse ciclo (BRASIL, 2015a).

A hemovigilância é conceituada como as ações de vigilância nas etapas do ciclo do sangue. Seu principal objetivo é identificar os eventos adversos, intervir em suas causas, melhorando a qualidade dos processos, produtos e a segurança para o doador e receptor. Durante o processo transfusional, realizado nos hospitais, a hemovigilância é realizada pelo serviço de gerência de risco (BRASIL, 2015b; GRANDI JL, et al., 2018).

Existem profissionais que acreditam que as notificações têm caráter punitivo. No entanto, este entendimento contrapõe-se ao preconizado pelo marco teórico e operacional da hemovigilância, que direciona medidas corretivas, educativas e preventivas adequadas aos casos de eventos adversos durante o processo transfusional (BRASIL, 2015b).

De acordo com o $7^{\circ}$ Boletim de Hemovigilância, no ano de 2014 a nível nacional houve 11.247 notificações de eventos adversos relacionados ao ciclo do sangue. Desse quantitativo, a região norte notificou 438 eventos (a menor taxa do Brasil) e o estado do Pará realizou 169 notificações (a maior taxa da região norte). Em relação aos anos anteriores, houve um aumento considerável das taxas de notificação, no entanto cabe aos gestores articularem estratégias com vistas à melhoria da qualidade transfusional e a redução da ocorrência de eventos adversos (BRASIL, 2015c).

Sendo assim, o processo transfusional é dividido com a fase pré-transfusional, transfusional e póstransfusional, tendo por base os critérios clínicos do paciente. Existem diversas normas e procedimentos preconizados cada etapa, tendo como finalidade garantir a qualidade do procedimento e prevenir erros que podem comprometer a saúde e o bem-estar do paciente. (BRASIL, 2015a).

Nesse sentido, existem diversos instrumentos que podem ser utilizados para o planejamento, monitoramento e controle das boas práticas em hemoterapia. A exemplo disso, temos os instrumentos da 
gestão de qualidade, como: Checklist, diagrama de Ishikawa, 5W2H (plano de ação), PDCA (planejar, fazer, verificar e agir), Brainstorming (tempestade de ideias) e outros; os quais podem auxiliar a hemovigilância, fornecendo ao gestor subsídios para intervir e melhorar os processos de trabalho, e também minimizar os eventos adversos no processo transfusional (BRASIL, 2015b; GALDINO SV, et al., 2016).

Dessas ferramentas, destaca-se o diagrama de Ishikawa, que permite identificar as possíveis causas que determinam um problema específico. A sua execução divide-se em 5 etapas: definição do problema, estruturação do diagrama, agrupamento de informações, classificação das causas e conclusão do diagrama. $\mathrm{E}$, destaca-se, também, o $5 \mathrm{~W} 2 \mathrm{H}$ que é plano de ação, este visa organizar a maneira de intervir em um determinado problema (BEZERRA F, 2014).

A utilização dessas ferramentas pela gestão do serviço de hemoterapia permite melhorar a qualidade e resolutividade de problemas do serviço. A educação permanente é uma ação estratégica para a melhoria dos processos, pois visa gerar discussões e questionamentos, com base no conhecimento científico, quanto aos problemas enfrentados na realidade da gestão do cuidado e dialogar quanto ao aperfeiçoamento do serviço (COSTA MAR, et al., 2018).

Desse modo, este estudo tem por objetivo descrever a utilização do $5 \mathrm{~W} 2 \mathrm{H}$ (plano de ação) e do diagrama de Ishikawa como mecanismos para a melhoria das notificações em hemovigilância e, consequentemente, da qualidade do processo transfusional.

\section{DETALHAMENTO DA EXPERIÊNCIA}

Trata-se de um relato de experiência, desenvolvido a partir da vivência de acadêmicos do curso de Enfermagem da Universidade do Estado do Pará (UEPA). O estudo seguiu as etapas da metodologia da problematização do arco de Charles Maguerez (BERBEL NAN, 2012).

O arco de Maguerez é constituído de 5 etapas: observação da realidade, que é a problemática encontrada no campo prático, sendo o ponto de partida do estudo; levantamento de pontos-chave, onde são escolhidos os temas de maior relevância como representação da realidade a ser trabalhada; teorização, que consiste na fundamentação da temática escolhida; hipóteses de solução, proveniente da busca de possíveis soluções, criativas e críticas, do problema apresentado; e aplicação à realidade que seria o retorno ao campo prático com as possíveis soluções encontradas, na tentativa de transformar a questão problema (MELO MC, et al., 2016; BERBEL NAN, 2012).).

A experiência foi desenvolvida em um Centro de Alta Complexidade em Oncologia (CACON), sendo um hospital referência em Oncologia, Nefrologia, Neurologia e Transplante na região norte, nos quais são desenvolvidas atividades de assistência, ensino e pesquisa.

Na etapa de observação foi realizada uma visita técnica à Gerência de Risco do hospital, que desenvolve o papel de tecnovigilância, farmacovigilância, hemovigilância e Segurança do Paciente. No decorrer dos relatos da equipe da Gerência de Risco, foi observado como problemática a subnotificação e as notificações incorretas dos eventos adversos relacionados a transfusão sanguínea.

Dessa forma surgiram os seguintes pontos-chave: A elevada ocorrência de transfusões sanguíneas realizadas no hospital e reduzida taxa de notificações de eventos adversos, assim como às fragilidades no preenchimento completo e adequado nos controles antes, durante e após o processo transfusional.

A partir dos pontos-chave encontrados, seguiu-se para a fase de teorização, na qual foi realizado o levantamento das evidências científicas acerca do ciclo do sangue e do processo de hemovigilância, gestão e ensino, segundo as regulamentações técnicas e legais. Além disso, a teorização se deu pela busca de artigos indexados na Biblioteca Virtual da Saúde (BVS), através dos seguintes Descritores em Ciência da Saúde (DeCS): Hemovigilância, Gestão em Saúde e Educação Permanente. A busca foi realizada com o auxílio do boleador de pesquisa "AND". Os únicos resultados encontrados foram referentes a combinação de "Hemovigilância" and "Educação permanente", na qual obteve-se dois artigos. Um indexado na PubMed em 
2015 e indisponível para download gratuito e o outro, indexado na base de dados LILACS, em 2009 com texto completo e gratuito.

Diante dos fatores contribuintes identificados para o problema e com base na teorização, foram elaboradas propostas de melhorias distribuídas em dois momentos. O primeiro por meio da utilização da ferramenta $5 W 2 H$ (plano de ação) como instrumento de promoção de educação permanente, e o segundo, a utilização da proposta do diagrama de Ishikawa a ser utilizado pela equipe clínica como ferramenta de gestão para identificar as causas acerca da subnotificação. A partir desta metodologia, foi possível promover a identificação, pela equipe clínica, dos fatores contribuintes para o problema, bem como o apontamento de medidas de melhoria a serem adotadas durante a rotina no processo transfusional.

Para auxiliar na atividade de educação continuada do primeiro momento, os pesquisadores construíram um cartaz explicativo, voltado aos profissionais do serviço, contendo o conceito de hemovigilância, seu objetivo, os eventos adversos passíveis de notificação e a conduta institucional diante do processo transfusional.

A ação educativa organizada pelos pesquisadores e a Agência Transfusional, e foi realizada nos locais com maior quantitativo de transfusões: Unidade de Atendimento Imediato (UAI), Clínica de Cuidados Paliativos Oncológicos (CCPO), Centro de Terapia Intensiva (CTI), clínica de hematologia oncológica, "hospital dia" e Bloco Cirúrgico.

Nos locais visitados, os pesquisadores abordaram os profissionais explicando acerca da importância da notificação dos eventos adversos relacionados à transfusão, o carácter educativo e a importância dessa prática para a melhoria e a segurança nos processos transfusionais. Ao final, foi entregue o cartaz aos profissionais como produto da ação realizada.

O segundo momento citado, se deu pelo retorno ao hospital, para a utilização da proposta do diagrama de Ishikawa como ferramenta de identificação das causas raiz que interferem na rotina de notificação e para levantamento das estratégias de que visam aumentar o quantitativo de notificações.

O diagrama de Ishikawa foi aplicado por meio de um diálogo, no qual se lançava a temática da hemovigilância e os profissionais relatavam suas experiências informalmente, a medida que o diálogo foi se desenvolvendo os pesquisadores começaram a identificar na fala das profissionais as possíveis causas que interferem na rotina de uma notificação de qualidade e o que poderia ser realizado para melhorar tal realidade.

O diálogo ocorreu com duas enfermeiras gerentes, de clínicas que realizam um grande quantitativo de transfusões, sendo essas: a clínica de hematologia oncológica e o "hospital dia". Cabe ressaltar que tais locais foram escolhidos por serem clínicas com pacientes mais estabilizados e com pouca ocorrência de situações de urgência nos processos de trabalho, além da disponibilidade da enfermeira gerente em participar do momento proposto representando os profissionais de sua clínica.

\section{DISCUSSÃO}

O cartaz utilizado nas clínicas, como forma de apresentação da temática, foi um produto entregue aos profissionais representando o retorno material ao serviço de saúde. Com ele, visou-se a divulgação e o acesso das informações sob a forma de conteúdo de fácil entendimento e visualmente mais atrativo acerca da hemovigilância.

No momento da ação, foi perceptível a boa receptividade dos profissionais quanto ao conteúdo abordado nas discussões que emergiram a partir do problema exposto pelos pesquisadores. Apesar da rotina agitada e da elevada demanda de procedimentos a serem feitos, a experiência quanto à utilização de ferramentas da gestão da qualidade na culminância da ação de educação permanente, foi salutar, pois possibilitou participação ativa dos profissionais.

Antes da utilização da adaptação do diagrama de Ishikawa, utilizou-se a ferramenta Brainstorming para contextualizar os profissionais e facilitar o processo. A partir dessa técnica, os pesquisadores apresentaram

REAS/EJCH | Vol. 11 (10) | e642 | DOI: https://doi.org/10.25248/reas.e642.2019 Página 4 de $\mathbf{7}$ 
o tema, definiram o objetivo a ser conquistado e as contribuições a serem dadas por meio do diagrama de Ishikawa. Houve a fase criativa (fase de apresentação do maior número de ideias coletivamente, sem análise crítica) e a fase crítica (os participantes defendem e analisam suas ideias individualmente) (NOBREGA MM, et al., 1997).

A partir da Brainstorming foi possível identificar, na fala dos profissionais, os motivos da baixa notificação e da notificação incorreta dos eventos adversos, e também as consequências relacionadas a esses problemas. Ademais, também se discutiu acerca de ações estratégicas que poderiam ser desenvolvidas para melhorar o processo de notificação. Com isso, os pesquisadores avaliaram a ação desenvolvida construindo seu próprio diagrama de Ishikawa, isso os possibilitou visualizar onde estavam os pontos críticos e suas soluções, conforme demonstra Quadro 1.

Quadro 1 - Causas da baixa notificação e notificação incorreta dos eventos adversos da transfusão e suas respectivas ações preventivas e corretivas.

\begin{tabular}{|c|c|c|c|}
\hline $\mathbf{N}$ & Causas & $\mathbf{N}$ & Ações \\
\hline 1 & Desconhecimento da importância da notificação & 1 & Educação em saúde \\
\hline 2 & Medo da punição & 2 & Conscientização do profissional \\
\hline 3 & Sobrecarga de trabalho & 3 & Melhoria no dimensionamento da equipe \\
\hline 4 & $\begin{array}{l}\text { Falha no retorno dos dados notificados para a } \\
\text { hemovigilância em forma de indicadores para a } \\
\text { clínica }\end{array}$ & 4 & $\begin{array}{l}\text { Sensibilização dos setores de gestão } \\
\text { para proporcionar feedback entre serviço } \\
\text { e resultados }\end{array}$ \\
\hline 5 & Longa jornada de trabalho & 5 & $\begin{array}{l}\text { Utilização de novas metodologias nas } \\
\text { capacitações, incluindo as metodologias } \\
\text { ativas }\end{array}$ \\
\hline 6 & Falta de comprometimento; & 6 & Realização de eventos pela instituição \\
\hline 7 & Falta de adequação às normas ins & 7 & $\begin{array}{l}\text { Liberação da equipe para os } \\
\text { treinamentos }\end{array}$ \\
\hline
\end{tabular}

Fonte: Diário de campo dos pesquisadores, 2018.

Desse modo, é possível estabelecer relações entre algumas causas apresentadas e ações estratégicas. Um fator que se destacou na ação desenvolvida foi a indicação, por parte dos profissionais, das metodologias ativas nas capacitações, de modo a fomentar uma aprendizagem significativa para a equipe. De acordo com Paiva MRF, et al. (2016), as metodologias ativas rompem com o modelo tradicional de ensino e fundamentamse numa prática problematizadora, voltada à realidade dos profissionais da saúde, incentivando-os a ter autonomia e compreender a importância de diversos processos.

A compreensão das causas e a execução de ações estratégicas é um fator gera uma consequência na qualidade e na segurança dos processos transfusionais. Pois, a cada 1000 transfusões são esperadas de 1 a 7 reações transfusionais, demonstrando que os eventos adversos irão ocorrer, sobretudo em paciente politransfundidos. Nesse sentido, a subnotificação acaba interferindo e acarretando riscos à saúde do paciente e afeta a atuação do gestor, de modo que este não consegue visualizar as reais causas do problema e as medidas necessárias para sua superação ou minimização (SILVA EM, 2015).

Em um estudo realizado em São Paulo, acerca das notificações em hemovigilância, foi identificado que geralmente os profissionais negligenciam as reações transfusionais leves (Evento adverso na hemotransfusão) por carência de educação continuada e educação permanente (GRANDI JL, 2018). O que corrobora com os dados de Dias MAM (2009), que identificou a pouca discussão sobre o referido tema e ressaltou a grande sobrecarga dos profissionais em sua rotina de trabalho. Fatores esses que também foram apontados pelas enfermeiras gerentes durante a ação educativa.

Portanto, o diagrama de Ishikawa permite ao gestor discutir de maneira mais ampla as causas que interferem no processo de notificação em hemovigilância de modo a ajudar tanto os gestores quanto os 
profissionais a refletirem sobre a criação e utilização de ferramentas que auxiliem e facilitem a realização da notificação e atuação diante dos eventos adversos na transfusão de sangue.

Em relação as limitações encontradas na realização da atividade desenvolvida, pode-se citar a dificuldade em obter a adesão de um quantitativo significativo de profissionais em um mesmo ambiente. Outro ponto limitante consistiu no curto período para explanar uma temática extremamente complexa e abrangente como o ciclo do sangue, eventos adversos e hemovigilância, implicando dessa forma em uma abordagem superficial do problema encontrado.

Nesse sentido, ressalta-se que a educação permanente é uma ferramenta com enorme potencial frente à melhoria da qualidade do cuidado e deve estar centrada em toda a equipe de saúde, especialmente a de enfermagem, visto que tanto o enfermeiro, como o técnico de enfermagem, prestam cuidados prolongados a pacientes internados em variadas unidades de atendimento e com isso todos necessitam estar treinados e atualizados em relação aos procedimentos mais simples e mais complexos (CRUZ ENN, CARRIJO AR, 2017).

Estas ferramentas são alternativas educacionais que viabilizam o desenvolvimento de ações voltadas à aprendizagem significativa em meio aos processos de trabalho, assumindo dessa maneira um caráter de potenciais transformadoras das práticas em saúde, de forma participativa e contextualizada. Logo, não basta apenas 'saber' ou 'fazer', é preciso 'saber fazer' com base nas evidências científicas, garantindo assim o comprometimento profissional com a qualidade do serviço oferecido aos pacientes (AMARO MOF, et al., 2018).

\section{CONCLUSÃO}

O presente trabalho permitiu ressaltar a importância da integração entre gestão, ensino e assistência. O serviço ganhou com o engajamento dos acadêmicos que trouxeram uma visão exterior à sua rotina e que se comprometeram em melhorar a qualidade da assistência; ao mesmo tempo, essa integração foi positiva para a formação dos acadêmicos, pois estimulou a capacidade de resolução de problemas da vida profissional. A subnotificação relacionada à hemovigilância é uma relevante questão a ser trabalhada nos hospitais. A notificação é o principal instrumento que garante o bom desempenho das atividades da gerência de risco e que se for negligenciada representa um obstáculo na promoção da segurança do paciente. Isso, principalmente no contexto de transfusão sanguínea, um procedimento complexo que envolve questões culturais, riscos de infecções ou outras reações, além de necessidade de uma estruturada rede de vigilância por meio de efetivas atividades de gestão e gerenciamento. Portanto, a importância da cultura de notificação precisa ser estimulada pela gestão por meio da educação permanente. E, além disso, este estudo evidencia o papel do enfermeiro como profissional que ao mesmo tempo domina as ferramentas e os conhecimentos da gestão e que também vivencia a realidade da assistência; sendo capaz de identificar as causas da subnotificação e buscar meios para amenizar essa situação.

\section{REFERÊNCIAS}

1. AMARO MOF, et al. Concepções e Práticas dos Enfermeiros Sobre Educação Permanente no Ambiente Hospitalar. Arquivos de Ciências da Saúde da UNIPAR. 2018; 22(2): 81-94.

2. BERBEL NAN. A metodologia da problematização em três versões no contexto da didática e da formação dos professores. Revista Diálogo Educacional, 2012; 12 (35): 103-120.

3. BEZERRA F. 2014. In: Diagrama de ishikawa: princípio da causa e efeito. Disponível em: http://www.portaladministracao.com/2014/08/diagrama-de-ishikawa-causa-e-efeito.html. Acesso em: 20 nov. 2018.

4. BRASIL. Ministério da saúde. Guia para uso de hemocomponentes. 2.ed. Brasília: Ministério da Saúde; 2015a; $136 \mathrm{p}$. 
5. BRASIL. Ministério da Saúde. Marco conceitual e operacional de hemovigilância: guia para hemovigilância no Brasil. 1.ed. Brasília: Ministério da saúde, 2015b; 77 p.

6. BRASIL. Ministério da Saúde. Boletim de hemovigilância no7. 7.ed. Brasília: Ministério da saúde, $2015 \mathrm{c} ; 14$ p.

7. COSTA MAR. Educação permanente em saúde: a concepção freireana como subsídio à gestão do cuidado /Permanenteducation in health. Revista cuidado é fundamental (Online). 2018; 10(2): 558-564.

8. CRUZ ENN, CARRIJO AR. Processo de Educação Permanente em um Hospital Público: Percepção de Enfermeiros Gestores. Revista Saúde e Desenvolvimento, 2017;11(6): 121- 136.

9. DIAS MAM. O enfermeiro na hemovigilância: sua formação e competências. Dissertação (Mestrado em Enfermagem) - Escola de Enfermagem Anna Nery da Universidade Federal do Rio de Janeiro, Rio de Janeiro, 2009; $174 \mathrm{p}$.

10. GALDINO SV, et al. Ferramentas de qualidade na gestão dos serviços de saúde: revisão integrativa de literatura. Revista Eletrônica Gestão e Saúde, 2016; 7(supl. 1):1023-1057.

11. GRANDI JL, et al. Hemovigilância: a experiência da notificação de reações transfusionais em Hospital Universitário. Revista da Escola de Enfermagem da USP, 2018; 52:1-7.

12. MELO MC, et al. Aprendizagem baseada na problematização:utilizando o arco de Maguerez na graduação em enfermagem. Revista eletrônica Gestão e Saúde. (Online), 2016; 7(1): 247-259.

13. NOBREGA MM, et al. Uso da técnica de brainstorming para tomada de decisões na equipe de enfermagem de saúde pública. Revista brasileira de enfermagem, 1997; 50(2):247-256.

14. PAIVA MRF, et al. Parente JRF, Brandão IR, Queiroz AHB. Metodologias ativas de ensino-aprendizagem: revisão integrativa. Sanare (Sobral, Online), 2016; 15(2): 145-153.

15. SILVA EM. Ocorrência de reações transfusionais em um hospital público na cidade do recife. Monografia (Especialização em Hematologia) - Universidade Federal de Pernambuco, Recife, 2015; 50 p. 